\title{
Supporting deep understanding with emerging technologies in a STEM university math class
}

\author{
Domenico Brunetto, Clelia Marchionna, Elisabetta Repossi \\ Department of Mathematics, Politecnico di Milano, Italy.
}

\begin{abstract}
In this work we present an innovative learning environment format, based on student-centred activities, that may support undergraduate students to deep understanding mathematics in the first year of engineering degree. In particular, we refer to the difficulties students meet in the transition from the high school mathematics to the one they meet at university, which requires a significant shift to conceptual understanding, especially in Calculus courses. The goal of this presentation is to investigate the case of multivariable functions, a topic at the foundation of many mathematical models and its application. We show the results of the first pilot study which involves 160 undergraduate students. More precisely, we report how a flipped-learning approach based on online activities and working group allows students to deep understand the main properties concerning multivariable functions.
\end{abstract}

Keywords: Flipped learning; Calculus; First-year engineering degree; Multivariable functions; Matlab; Instructor videos. 


\section{Introduction}

Mathematics courses are pivotal in STEM programs; however, many scholars show that firstyear students face with difficulties (see, for instance, Gamer \& Gamer, 2001; Gueudet, 2008; Andrà, Bernardi \& Brunetto, 2019). The reasons of such difficulties are rooted in the differences between high school and university (Gueudet, 2008). For instance, student face a more conceptual mathematics at university (Clark \& Lovric, 2008), and they perceive different importance of the available resources at university compared with high-school (Kock, Brunetto \& Pepin, 2019).

In engineering programs calculus course are the most spreading, their syllabus is composed of many topics that may range from the foundations of mathematics to multivariable integration. The notion of a multivariable function is fundamental in mathematics and its applications; Martínez-Planell and Trigueros-Gaisman (2012) show that students need more opportunities to reflect on actions related to the construction of the domain, range and graphs of a rich diversity of functions and the transformations among representation registers. Hence, we argue that multivariate function has a strong conceptual nature. However, students are used to viewing only the procedural one, such as the integration rules, which is a not neglectable aspect of this topic. Therefore, the dual nature of the topic poses specific challenges to the students. On the path of Andrà and et al. (2019), in this work we address the problem to support the move from procedural to conceptual approaches to mathematics in first-year calculus courses, focusing on multivariable functions.

To that end, we agree with Gamer and Gamer (2001) who show that student-directed learning promotes conceptual learning more effectively if compared to teacher-directed approach. In particular, we resort to relative new flipped-learning formats (Bergmann \& Sams, 2015) that encourages student-directed learning.

The presentation of this work unfolds as follows. Firstly, we briefly recall the main students' difficulties with the mathematical content. Then, in Section 3 and 4, we describe how we designed and carried out a student-centred activity in a first-year calculus course for Environmental Engineering students at Politecnico di Milano. In Section 5, we report the data analysis, and in Section 6 we briefly discuss the main results and report the conclusions.

\section{Mathematical content: students' difficulties with multivariate functions}

Every high school student has experienced functions of a single independent variable, that is $y=f(x)$, where $x$ and $y$ are the independent and dependent variables, respectively. Moreover, many students of the first calculus course know how differentiating and integrating such functions work. Furthermore, the graphical representation is generally a beneficial register for understanding all the conceptual concepts. For instance, the differentiation is related to 
the tangent of the function $f(x)$ while the integration concerns the area bounded by the function.

However, almost all the functions that model phenomena in nature do not depend on a single independent variable but is usually determined by two, three or more independent variables. Thus, for example, the volume $(V)$ of an ideal gas depends on the pressure $(p)$, and the temperature $(T)$, therefore, the volume is a two-variable function, that is $V=f(p, T)$.

Jones and Dorko (2015) provide evidence that the students' conceptualizations of multivariate function (e.g., the boundary volume) are rooted in and connected to prior understandings of single-variable function. However, students demonstrate a very limited understanding of two-variable functions and its geometrical interpretation (Martínez-Planell \& Trigueros-Gaisman, 2012).

According to the above authors, instructors use to present multivariate functions starting from the case of only two-variables, e.g., $x$ and $y$ and provide the graph of a function $z=f(x, y)$ as a surface in a Cartesian reference system in the space $(x, y, z)$. Then, exploiting graphical register, the main conceptual aspects are developed. However, the object representations, both the three-dimensional surface on two-dimensional "canvas", and the two-dimension contour map of $z=f(x, y)$ are all but easy. Moreover, even the former representation is easily readable only if a student has understood the underlying three-dimensional object.

\section{Research methods}

\subsection{The context of the research}

In this work, we consider the data collected during the first-year course named "Mathematical Analysis and Geometry" (MAG), in particular, the "Matlab course" that supports the mathematical understanding. The whole course is a 1-year long mathematics course (September-June) devoted to Environmental Engineering students. The total number of students enrolled in each year is about 200, with $30 \%$ of female students (for instance, in 2019,57 are female and 139 are male) the most majority of them is 20 yo. Another interesting aspect of such course is that, the $30 \%$ of students enrolled in the course has low level of mathematical skill because of these students performed bad at entrance test (score $<60 \mathrm{pt}$ over 100). Such data informs us about their potential difficulties with the mathematical contents and in particular the multivariate functions.

The Matlab course, composed of six laboratories sessions (two hours each), aim at supporting the deep understanding of the basic concepts of mathematical analysis and linear algebra. The software Matlab, produced by Mathworks (www.mathworks.com), is worldwide used both in academic and in professional fields, such as data science and scientific modelling. 
We proposed the implementation of some math procedures related to the syllabus of the math course encouraging the usage of its graphical toolbox to prompt students in the deep conceptual thinking of the topics.

\subsection{The new innovative learning environment}

The structure of the Matlab course was unchanged from three academic years but in the last a.y. (September 2018-June 2019) we adopted the flipped learning methodology. In particular, we set a new learning environment composed of online asynchronous instructional videos and online quizzes with instant feedback as the "out-of-class" part in the flipped learning, and in-presence group work activities as the "in-class" part (Bergmann \& Sams, 2015).

More precisely, before each of the six in-presence parts, students were invited to watch an instructional video (10-15 minutes long) and to answer a Google form in order to self-assess their learning. The instructional videos have been recorded by the professor of the Matlab course (one of the authors) using Screencast-O-Matic tools (screencast-o-matic.com) and has been posted on YouTube. While the Google form is composed of multiple-choice questions about both the Matlab commands and the relative context (e.g. Which of the following commands would you use if you want to clear that specific variable?).

In each meeting, the professor shows and comments the quizzes results, then students are asked to work in small groups. After that, lesson ends with a class discussion and followed by a teacher-directed moment, where the professor provided further insights about the mathematical content, its applications and the Matlab commands.

\subsection{Data collected}

The data of this work is composed of students' attendance in the Matlab course, the mark of the students both in the Matlab test and the MAG exam.

The Matlab course attendance is certificated by the answers to the google form and the presence in the laboratory. Those students, who have a more than $70 \%$ of attendance, gain the Matlab certificate. Such certificate allows students to make a non-compulsory Matlab test, gaining extra-bonus (up to 2 points) on the final MAG exam.

Moreover, at the end of the course, we proposed a satisfaction questionnaire about the new learning environment of Matlab course, 58 answers were collected. Only two questions are strictly related to the issue of this work: Q1 concerns what they like/dislike while Q2 ask for general comments.

Finally, we can compare the Matlab test marks of the cohort of students 2018/19 (C2019) with the cohort of the three previous academic years 2017/18 (C2018), 2016/17 (C2017) and 2015/16 (C2016) when the Matlab course was not delivered as "flipped". 


\subsection{Research question}

To recall, this work deals with the two-variables functions, a mathematical topic that is not well studied in the literature, but it is relevant due to two main reasons: on the one hand, the topic is at the centre of many applications in modelling natural and social phenomena, on the other hand, students have difficulties in dealing with such topic.

Hence, our research questions are: RQ1) How can we support the conceptual learning of twovariables functions? RQ2) To what extent the new innovative learning may support the deep understanding of two-variables functions?

\section{The lesson on two-variables functions}

In order to answer RQ1, we designed a lesson about the two-variables functions within the context of the new learning environment described above. This lesson was the fifth (over six) delivered in the Matlab course. The main goal was the representation and interpretation of three-dimensional mathematical objects.

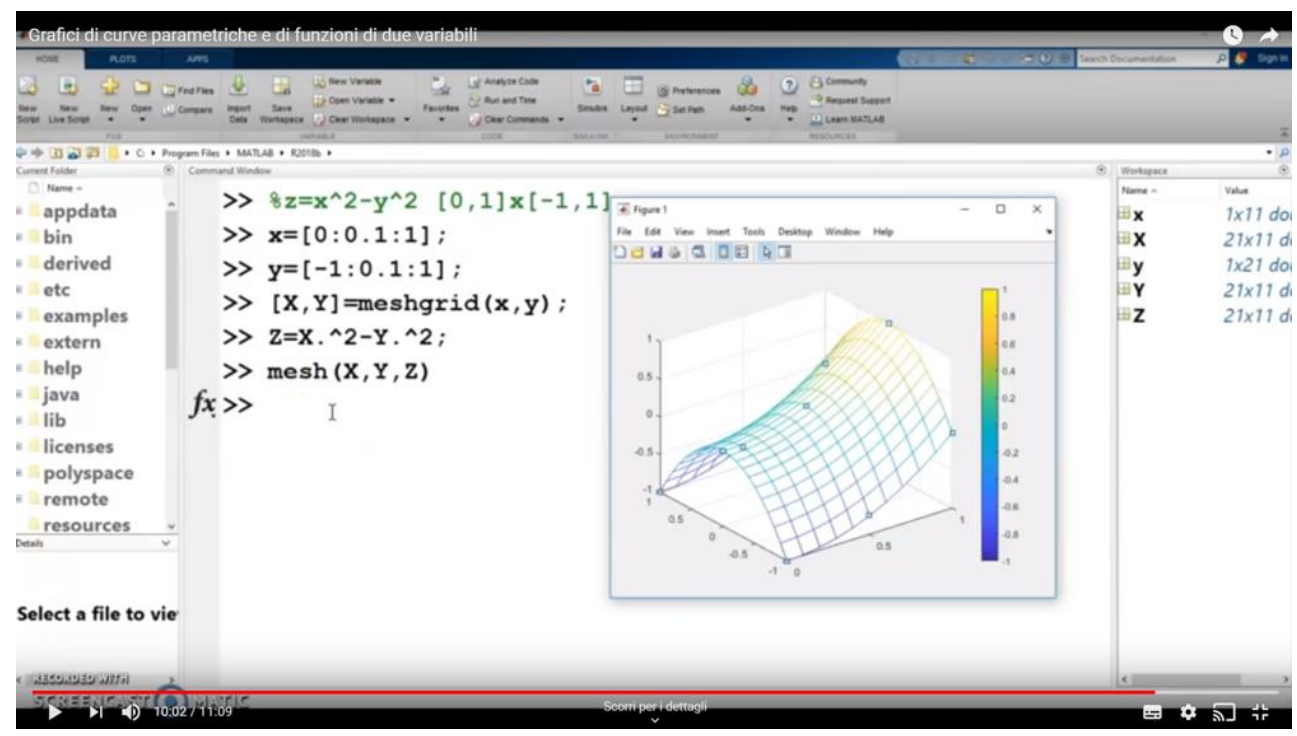

Figure 1. Overview of the instructional video about three-dimensional graph of a two-variable function $z=x 2-y 2$ using the command mesh. Source: authors, using Matlab and Screencast-O-Matic.

In the video, the instructor presents an exercise, for instance, "represent the graph of $z=x^{2}$ $y^{2}$ " (see the green text in Figure 1). Then the task is unfolded step by step, presenting the new commands and remarking the mathematical aspect. Finally, once the graph is shown, comments about mathematics and coding were given to make it more effective. 
Moving on the online quizzes, we used google form with the quizzes options. The answers of the students allowed to argue that a good understanding of the topic is achieved since the average score was 6.32 out 8 . However, some issues emerged: one is related to the use of a new tool, the so-called "instrumental genesis" (see, for instance, Rabardel, 1995), the other one concerns the conceptualization of the domain and its sampling.

The in-presence lesson was designed from the issues mentioned above. More precisely, at the beginning of the session, the instructor comments about the answers and addressed the issues. As regards the issue about the domain, she reported the lecture notes that accompany the course and clarified the use of the sample in terms of density. Then, students were invited to work in groups to solve three kind of tasks act to clarify the issues manipulating the mathematical objects: 1) direct application of procedures shown in the videos; 2) graphical representations in which the choice of the grid was crucial; and 3) integration of both mathematical content and software tool (e.g., calculation of a tangent plane).

During the lesson, the instructor noticed that students have watched the video, read lectures notes and knew the commands. At the end, the professor addressed the main difficulties and misconceptions, avoiding to provide solutions for the tasks because students were expected to compare and discuss within and among groups.

\section{Data analysis and results}

In order to address RQ2, in this section we report the data relative to students' performance and their answer to the satisfaction questionnaire relative to the C2019.

The average mark to the Matlab test of the students in C2019 is 24.19 (out of 30), which is better than the previous years since in C2017 is 22.37 and the others are below 20/30 (18.55 in C2018 and 19.96 in C2016). The employed ANOVA test shows that the four cohorts are statistical different (p-value $\leq 1 \mathrm{e}-6$ ), in particular C2019 strongly differs from C2018 and C2016 (p-value<1e-6) and weakly from C2017 (p-value=0.05). Looking closer at the marks distribution, see Table 1, we observe that the percentage of students who gain the lowest levels in C2019 is quite different to C2017, while those who gain the highest levels (A and B) is slightly the same in C2019 and C2017. We can argue that a shift of the performance occurs from the lowest level (E and D) to the medium one $(C)$. 
Table 1. Percentage of students divided into five levels. The distribution concerns the Matlab tests related to the four years under investigation.

\begin{tabular}{ccccc}
\hline & C2019 & C2018 & C2017 & C2016 \\
\hline Level E: Mark $\leq 14$ & $5.68 \%$ & $32.95 \%$ & $18.75 \%$ & $23.76 \%$ \\
Level D: $15 \leq$ Mark $\leq 17$ & $10.23 \%$ & $9.09 \%$ & $5.21 \%$ & $13.86 \%$ \\
Level C: $18 \leq$ Mark $\leq 23$ & $23.86 \%$ & $23.86 \%$ & $17.71 \%$ & $26.73 \%$ \\
Level B: $24 \leq$ Mark $\leq 29$ & $48.86 \%$ & $28.41 \%$ & $47.92 \%$ & $25.74 \%$ \\
Level A: Mark=30 & $11.36 \%$ & $5.68 \%$ & $10.42 \%$ & $9.90 \%$ \\
\hline
\end{tabular}

Source: Authors (2020).

Now we focus on the two tasks of the Matlab test (Task 5 and Task 6) that refer to threedimensional representation of a function $\mathrm{z}=f(x, y)$. The two tasks aim at prompting students in conceptual understanding because they require students to distinguish maxima/minima (along $z$-axis) from the range of the domain (along $x$-axis and $y$-axis). Moreover, the tasks require to distinguish and compare between global and local maxima/minima, and more generally between open and close sets. The students' marks in these two tasks along the last four years reveal that in $\mathrm{C} 2019$ students perform better for both tasks. Indeed, the average marks for Task 5 (max 5pts) were 3.62 in C2019, 2.57 in C2018, 3.5 in C2017 and 2.46 in C2016. Similar behavior for Task 6 (max 5pts): 3.59 (C2019), 2.52 (C2018), 3.36 (C2017) and 2.89 (C2016).

As regards the MAG test, we consider the exams passed by September 2019, and the relative exam mark averages. The number of students enrolled in C2019 MAG course is 196, and 99 (about 50\%) students passed the MAG exam with an average mark of 25.9 out of 30 . However, the number of Matlab certificate obtained is 110 , among them 81 students (about $74 \%$ ) have passed the MAG exam with an average mark of 26.5. We can infer that 18 (9981) students have passed the exam but have not gain the Matlab certificate, those 18 students' average mark is worst (23.2/30). Since the difference is about 3 points, can we conclude that the new learning environment for the Matlab course had a reinforcing impact? Firstly, we recall that the MAG grade is influenced by the extra-bonus due to the Matlab test, which contributes for just one point on average in MAG grade C2019. Therefore, we can infer a good mark in Matlab test influences the performance in MAG exam. Moreover, in the previous year 2017/2018, attendance at Matlab course was far lower and, even though the percentage of students who passed the exam by September (C2018) was exactly the same (about 50\%), their final grade average was 23.90 , exactly two points lower.

Finally, we report the most significant answer related to questions of the satisfaction questionnaire. Q1 was about what students liked most about the course: the $38 \%$ of the 
students liked the new approach (e.g., work in groups during the in-class moment, and the link between the software and the mathematical content); the $36 \%$ liked the content, in particular, visualizing three dimensional objects and to have a deeper understanding of twovariable functions; the other appreciated the materials of the course (videos, lecture notes, online quizzes). Finally, answering Q2, most of the students would have suggested the course to their friends, because they appreciate the Matlab course and a software itself as an instrument to support their studies more in depth.

\section{Conclusions}

The focus of our research is on how to support the students' conceptual understanding of two-variable functions (RQ1) and to what extent this support can influence the conceptual understanding (RQ2). In particular, we are interested in developing a deep understanding of the topic resorting graphical representations using Matlab software. To this end, we designed a new learning environment according to the flipped learning methodology. The results of our investigation reveal that the new learning environment, that is the group work activities during the in-class moment paired with the instructional video and the quizzes, reached the goal of supporting students to the conceptual learning of two-variables functions. More precisely, the out-of-class moment was effective both in activating the students thinking and in sharing an instrument that the professor and students know and can use (Andrà et al., 2019). Moreover, the possibility to gain extra points for the exams prompted students in engaging the out-of-class activities and in attending the in-class moment, that effect their conceptual knowledge of the topics. In this way, emergent technologies sustain the students' deep understanding of a challenging topic. Finally, we conclude adding that this approach can be used in other STEM topics with the appropriate adjustment.

\section{References}

Andrà, C., Bernardi, G., \& Brunetto, D. (2019). Teaching with emerging technologies in a STEM university math class. In 5th International Conference on Higher Education Advances (HEAd'19) (pp. 963-971). 2019, Editorial Universitat Politècnica de València.

Bergmann, J., \& Sams, A. (2015). Flipped learning for math instruction (Vol. 2). International Society for Technology in Education.

Clark, M., \& Lovric, M. (2008). Suggestion for a theoretical model for secondary-tertiary transition in mathematics. Mathematics Education Research Journal, 20(2), 25-37.

Gamer, B. E., \& Gamer, L. E. (2001). Retention of concepts and skills in traditional and reformed applied calculus. Mathematics Education Research Journal, 13(3), 165-184.

Gueudet, G. (2008). Investigating the secondary-tertiary transition. Educational studies in mathematics, 67(3), 237-254. 
Jones, S. R. \& Dorko, A. (2015). Students' generalizations of single-variable conceptions of the definite integral to multivariate conceptions. In (Eds.) T. Fukawa-Connelly, N. Engelke Infante, K. Keene, and M. Zandieh, Proceedings of the 18th Annual Conference on Research in Undergraduate Mathematics Education, Pittsburgh, Pennsylvania.

Kock, Z. J., Brunetto, D., \& Pepin, B. (2019). Students' choice and perceived importance of resources in first-year university calculus and linear algebra. In: proceedings of the 14th International Conference on Technology in Mathematics Teaching.

Martínez-Planell, R. \& Trigueros Gaisman, M. (2012). Students' understanding of the general notion of a function of two variables. Educational Studies in Mathematics, 81(3), 365384.

Rabardel, P. (1995). Les hommes et les technologies; approche cognitive des instruments contemporains. Armand Colin. 